
\title{
28 Research Suare \\ Thrombocytopenia in Pediatric Congenital Heart Diseases Patient with the Transcatheter Closure
}

\section{Lei Yang ( $\nabla$ yangleidr@163.com )}

Shanghai Children's Medical Center https://orcid.org/0000-0001-8463-1165

\section{Ying Guo}

Shanghai Children's Medical Center

Meirong Huang

Shanghai Children's Medical Center

\section{Xu Zhang}

Shanghai Children's Medical Center

Xinyi Xu

Shanghai Children's Medical Center

Wei Gao

Shanghai Children's Medical Center

Tingliang Liu

Shanghai Children's Medical Center

\section{Research article}

Keywords: thrombocytopenia, congenital heart diseases, the transcatheter closure

Posted Date: July 24th, 2020

DOI: https://doi.org/10.21203/rs.3.rs-47273/v1

License: (a) (i) This work is licensed under a Creative Commons Attribution 4.0 International License. Read Full License 


\section{Abstract}

Background: Postoperative thrombocytopenia with congenital heart disease (CHD) after percutaneous transcatheter closure (PTC) has seldom been reported. We sought to investigate the treatments, severity and causes of postoperative thrombocytopenia among patients with CHD undergoing PTC.

Methods: Six PTC patients undergoing postoperative thrombocytopenia in our department were included. Platelet count was measured at admission and daily after PTC at least for day 9.

Results: Three patients had platelet counts below $30 \times 10^{\wedge} 9 / \mathrm{L}$ while in the other two patients the counts were below $50 \times 10^{\wedge} 9 / \mathrm{L}$. All patients achieved a stable platelet count, ranging from $99 \times 10^{\wedge} 9 / \mathrm{L}$ to $241 \times 10^{\wedge} 9 / \mathrm{L}$ after day 9. The platelet counts recovered after being given intravenously the methylprednisolone (two patients), immunoglobulin (one patient) and platelet transfusions (two patients). During the whole course, the face and limbs of one patients were visible ecchymosis but no major bleeding events were recorded.

Conclusion: In this report, the clinical outcome of our patients were satisfactory; they complaints regressed after above treatment.

\section{Background}

Congenital heart disease (CHD) is a structural and functional heart disease, which is present at birth. Incidence of $\mathrm{CHD}$ is about $810 / 1000$ live births worldwide and varies with modern diagnostics. ${ }^{1}$ It can be associated with several short- and long-term complications and lead to significant mortality ${ }^{2}$. Children suffering from $\mathrm{CHD}$ have a more aggressive clinical course when compared with their adult counterparts ${ }^{3}$. The transcatheter closure of CHD including ventricular septal defect (VSD) and patent ductus arteriosus (PDA) is an effective and less invasive method as compared to the surgical ligation. In addition, it has been reported that approximately 6$12 \%$ CHD patients may develop postoperative complications of transcatheter closure within a few days to weeks, such as hemolysis, residual shunt, repatency ${ }^{4}$. In the present report, we encountered pediatric patients who suffered from postoperative thrombocytopenia after $\mathrm{CHD}$ occlusion.

\section{Materials And Methods}

Ethics approval was obtained from Medical School of Shanghai Jiaotong University for our study, which conformed to the principles of the Helsinski Declaration. A total of $7 \mathrm{CHD}$ patients who had undergone transcatheter CHD closure (TCC) at Shanghai Children's Medical Center, were screened and eligible patients were recruited into the study. Patients gave written informed consent prior to the TCC. Patient demographics and angiographic profiles were collected. The VSD patient received aspirin therapy prior to the operation.

Femoral arteriovenous catheterization

After general anesthesia after tracheal intubation, the femoral arteriovenous was punctured at the anterior wall with 21-gauge arterial needles through which a 0.018-inch straight floppy tip guidewire (20-cm length) was advanced upon appearance of smooth flow. Following this, the needle was withdrawn and a hydrophilic 4F or $5 F$ introducer sheath (11-cm length) with dilator length of $16 \mathrm{~cm}$ was inserted over the guidewire into the radial 
artery. Subsequently, the wire and dilator were removed. All patients had total procedural heparin $100 \mathrm{U} / \mathrm{kg}$ given as part of the TCC procedure. The occlusion was performed according to the routine of our hospital.

\section{Results}

Between September 2011 and August 2017, 6 patients (all females) were found to have experienced the declines in blood platelet counts after transcatheter VSD or PDA closure in the department of cardiology of our facility, and two of them requested emergency platelet transfusions. The median age of these patients was 13 months (range, 8-24 months). All patients had a VSD or PDA with a diameter larger than $3 \mathrm{~mm}$ (3-12 mm) and a left-to-right shunt, and one of them also had severe pulmonary hypertension, as determined by right heart catheterisation. After placing VSD or PDA occluders, four patients were found to a have a trivial residual shunt. The cardiovascular characteristics of the patient are detailed in Table 1. The nadir platelet counts invariably occurred ranged from the days 1 and 7 after the transcatheter closure procedure and three patients had platelet counts below $30 \times 10^{\wedge} 9 / \mathrm{L}$ while in the other two patients the counts were below $50 \times 10^{\wedge} 9 / \mathrm{L}$. All patients achieved a stable platelet count, ranging from $99 \times 10^{\wedge} 9 / \mathrm{L}$ to $241 \times 10^{\wedge} 9 / \mathrm{L}$ after day 9 (see Fig. 1). The platelet counts recovered after being given intravenously the methylprednisolone (two patients), immunoglobulin (one patient) and platelet transfusions (two patients). Based on the above clinical and laboratory findings, they were diagnosed with postoperative thrombocytopenia after CHD occlusion. During the whole course, the face and limbs of one patients were visible ecchymosis $\square$ see Fig. 2₫but no major bleeding events were recorded.

Table 1

Cardiovascular characteristics of patient with thrombocytopenia after transcatheter CHD closure.

\begin{tabular}{|c|c|c|c|c|c|c|c|}
\hline \multirow{2}{*}{$\begin{array}{l}\text { Gender/age } \\
\text { F/14 m }\end{array}$} & \multirow{2}{*}{$\begin{array}{r}\text { PDAVSD } \\
\text { diameter } \\
\text { PDA }\end{array}$} & \multicolumn{2}{|c|}{$\begin{array}{l}\text { PA pressure* } \\
(\mathrm{mmHg})\end{array}$} & \multicolumn{2}{|c|}{$\begin{array}{l}\text { AA pressure* } \\
(\mathrm{mmHg})\end{array}$} & Residual shunt & \multirow[t]{2}{*}{$\begin{array}{l}\text { Residual } \\
\text { shunt at } 6 \\
\text { hours }\end{array}$} \\
\hline & & $7 \mathrm{~mm}$ & $38 /$ & $3 / 30$ & $101 / 45 / 70$ & + & \\
\hline $\mathrm{F} / 12 \mathrm{~m}$ & PD/ & $3.1 \mathrm{~mm}$ & $61 /$ & $34 / 49$ & $111 / 62 / 89$ & + & + \\
\hline $\mathrm{F} / 9 \mathrm{~m}$ & PD/ & $3.7 \mathrm{~mm}$ & $41 /$ & $3 / 33$ & 104/53/80 & - & + \\
\hline $\mathrm{F} / 8 \mathrm{~m}$ & PD/ & $3 \mathrm{~mm}$ & $24 /$ & 10/17 & $100 / 60 / 52$ & - & - \\
\hline$F / 2 y$ & VSL & 12 mm & $35 /$ & $5 / 23$ & $99 / 47 / 63$ & + & + \\
\hline
\end{tabular}

Legend: CHD: patent ductus arteriosus; PA: pulmonary artery; AA: aortic artery; ${ }^{\mathrm{PA}} \mathrm{A}$ and $\mathrm{AA}$ pressures are shown as systolic/diastolic/mean.

\section{Discussion}

In this report, we have presented 6 unusual children who suffered from thrombocytopenia after transcatheter closure of VSD or PDA. Thrombocytopenia is a well-recorded complication after invasive procedures; the leading causes include internal bleeding, sepsis, and thrombosis ${ }^{5}$. The occluder mechanical factors may play an important role because both ventricular assist devices and cardiopulmonary bypass are associated with platelet consumption leading to thrombocytopenia too underlying that the mechanical factors may destroy the platelets especially the trivial residual shunt exsisited ${ }^{6}$. Given the endothelial injury and high flow velocity, the site of a residual shunt after $\mathrm{CHD}$ closure provides an ideal target for platelet adhesion and subsequent platelet plug 
formation, which could be a reasonable explanation for the decrease in the residual shunts seen in such a short period in the patient ${ }^{3}$. In this case, platelets play the most crucial role in the maintenance of haemostasis due to the "radial dispersion" effect. The patient presented a potential risk of a residual shunt after the transcatheter closure procedure and this harmed the platelets.

The more common view is that occluder-related thrombocytopenia (ORT) is the main cause of thrombocytopenia after closure. In recent years, with the development of minimally invasive technology, interventional treatment of CHD has developed by leaps and bounds. Now, the percutaneous closure, which is a CHD treatment, has been widely recognized in the world. The principle of the immediate effect of the occluder is that the grid-like fibrous patch attached to the metal basket is used as a matrix to induce thrombosis in order to completely block the intraductal blood flow. After blocking, a lot of platelets accumulate and are consumed at the interior and surface of the large occluder in a short time, while the platelets accumulated and protruded on the umbrella of descending aorta continue to be affected by high-speed blood flow within the aorta, which finally become fragments and are washed out. When thrombocytopenia is beyond the platelet's own regenerative capacity, the clinical manifestations are progressive ORT. As time goes on, platelets form relatively fixed thrombus. After the vascular endothelialization, the number of platelet loss begin to decrease; when the platelet production is more than the destruction, the number of platelets gradually returned to normal.

Besides, thrombocytopenia may be associated with residual shunt. The greater the shunt is, the higher the shunt flow rate is, the longer the shunt duration will be, and the more the platelets are consumed. With the end of the occluder, the residual shunt disappears, the platelets gradually return to normal. When there's no residual shunt, platelets can quickly adhere to the occluder metal mesh and its polyester mesh, and then platelets start exogenous coagulation pathways to form fixed thrombosis. The amount of platelet loss is small and does not cause a significant decline in platelets. In our research, 4 cases have residual shunt. The study also found that not all residual shunt occurred thrombocytopenia, which is related to the residual flow at the edge of the occluder. When blood spurts through the residual shunt, platelets were mechanically damaged by the metal mesh of the occluder. At the same time, in the high-speed blood flow erosion, platelets in the occluder formed fragments and were consumed a lot.

Some patients being allergic to occluder is a part of the reason why thrombocytopenia happens after CHD closure. The CHD occlude produced by Shanghai Shape Memory Alloy Materials Co., Ltd. has 3 layers of polyester film which are composed of polyester fiber. ORT may be related to the patient's resistance to polyester fiber in the polyester component of occluder. In addition, the impact of nickel ions on platelets should also be considered. The occluders are made of nickel-titanium alloy. Studies have suggested that nickel-containing medical materials have poor blood compatibility. The greater the dissolution of nickel ions is, the more seriously the platelets deform and aggregate. ORT is possibly caused because of a large number of platelets gathering in the occluder and forming thrombosis. However, the occluder used to block the atrial septal defect is often larger, but it has not been reported yet that ORT after intervention happens in patients with atrial septal defect, so the impact of nickel ions on children needs to be further studied.

In our hospital, the CHD patients received a heparin $(100 \mathrm{U} / \mathrm{kg})$ during the operation for anticoagulation which may cause the heparin-induced thrombocytopenia (HIT). HIT is usually divided into type 1 and type 2; type 1 is more common and a benign reaction, rather than immune response. It may be related to the direct activation of platelets caused by heparin. In type 1, platelet count was slightly reduced and can be self-recovery, usually no 
clinical manifestations. Type 2 represents an antibody mediated adverse drug reaction characterized by plateletactivating immunoglobulin $\mathrm{G}(\mathrm{IgG})$ that recognize platelet factor 4 (PF4)/heparin complexes and occurs in 0.2$0.5 \%$ of patients who are treated with heparin ${ }^{7}$. IgG/PF4/heparin complexes bind to platelet Fc receptors, activate the receptors and lead to thrombocytopenia. In the case of type 2 , any immunizing exposure to heparin, whether triggered by the first exposure to heparin or any subsequent re-exposure, leads to a relatively rapid decrease of the platelets which can result from a significant decline in platelet count 5 to 10 days after starting heparin therapy ${ }^{7}$. For the diagnosis of HIT (type 2), the HIT expert probability (HEP) score is accurate and well correlated with positive laboratory results. At a low HIT risk, further diagnostic procedures are not recommended and the heparin therapy is suggested. At a moderate and high HIT risk, the test for IgG against the heparin-PF4 comple is advised. Once HIT is suspected based on the clinical presentation, heparin anticoagulants and vitamin $\mathrm{K}$ antagonists (warfarin, acenocoumarol) should be immediately withdrawn and the supply of vitamin $\mathrm{K}$ is advisable. It is worth that direct thrombin inhibitors (DTIs; lepirudin, bivalirudin, argatroban) therapy may contribute to the HIT as they do not bind to blood platelets and are not neutralised by PF4. Last, but most important, factor Xa inhibitors should be administered whenever DTIs are not available. Intestinally, the HIT generally occurs after 5-10 days of heparin being given, while the occurrence of the thrombocytopenia in this study are within 3 days after the operation. The follow-up studies are needed to clarify this phenomenon.

The immune thrombocytopenia (IT)can also be another cause. Because the recovery is after the intravenous methylprednisolone and immunoglobulin, we speculated that an immune process might be the key underlying mechanism for thrombocytopenia in the patients. IT is a diagnosis of exclusion once other thrombocytoses such as reactive thrombocytosis, myelodysplastic syndrome (MDS), and other myeloproliferative neoplasms (MPN) have been eliminated. To date, IT is the most common cause of thrombocytopenia in children and can be considered as a possible manifestation of immunodeficiency, particularly common variable immunodeficiency, selective IgA deficiency, and DiGeorge's syndrome. Most IT have no exact etiology and portion can be caused by infectious diseases caused by HIV, HCV, Helicobacter pylori, and dengue virus, through different mechanisms, like molecular mimicry, modulation of the immune system's activity or suppression of bone marrow production. Corticosteroids alone or combined with immunoglobulin remain the first-line treatment for thrombocytopenia ${ }^{9}$.

\section{Conclusion}

In this report, the clinical outcome of our patients were satisfactory; they complaints regressed after above treatment. It is worth mentioning that no life-threatening bleeding occurred in the patient and that the thrombocytopenia, severe as it was, seemed to have been a self-limiting process. Therefore, the postoperative blood routine examination of the VSD or PDA patients should be more closely monitored.

\section{Abbreviations}

congenital heart disease (CHD)

percutaneous transcatheter closure (PTC)

ventricular septal defect (VSD)

patent ductus arteriosus (PDA) 
transcatheter CHD closure (TCC)

occluder-related thrombocytopenia (ORT)

heparin-induced thrombocytopenia (HIT)

immunoglobulin G (lgG)

recognize platelet factor 4 (PF4)

HIT expert probability (HEP)

direct thrombin inhibitors (DTIs)

immune thrombocytopenia $\mathbb{\text { ITT }}$

myelodysplastic syndrome (MDS)

myeloproliferative neoplasms (MPN)

\section{Declarations}

- $\quad$ Ethics approval and consent to participate囚Ethics approval was obtained from Medical School of Shanghai Jiaotong University for our study, which conformed to the principles of the Helsinski Declaration. A total of $7 \mathrm{CHD}$ patients who had undergone transcatheter CHD closure (TCC) at Shanghai Children's Medical Center, were screened and eligible patients were recruited into the study.

- $\quad$ Consent to publish \All authors are consent to publish.

- $\quad$ Availability of data and materials: Patients gave written informed consent prior to the TCC.

Competing interests : The authors declare no conflict of interest.

Funding: Not applicable

Authors' Contributions: Lei yang, Ying guo conceived and designed research; Meirong huang, Xu zhang, Xinyi Xu, Wei gao, Tingliang liu collected data and conducted research; Lei yang, Ying guo wrote the initial paper; Wei gao, Tingliang liu revised the paper; Lei yang, Ying guo had primary responsibility for final content. Lei yang, Ying guo take responsibility for all aspects of the reliability and freedom from the bias of the data presented and their discussed interpretation. All authors read and approved the final manuscript.

Acknowledgements: Not applicable

\section{References}

1. Shafi NA, Singh GD, Smith TW, Rogers JH. Sizing of patent ductus arteriosus in adults for transcatheter closure using the balloon pull-through technique. Catheter Cardiovasc Interv. 2017 Sep 25. 
2. Chen PC, Swanson JC, Masand P, Rodriguez JR, Mery CM. Diaphragmatic Hernia Associated With Absent Ductus Venosus and Anomalous Connection of an Obliterated Umbilical Vein to the Coronary Sinus. World J Pediatr Congenit Heart Surg. 2017 Jan;1:2150135117710927.

3. Wu B, Rong R. Thrombocytopenia in patients with patent ductus arteriosus after transcatheter closure: perilous complication or harmless incidental finding? Blood Transfus. 2013 Jul;11(3):477-478.

4. Stankowski T, Aboul-Hassan SS, Marczak J, Szymanska A, Augustyn C, Cichon R. Minimally invasive thoracoscopic closure versus thoracotomy in children with patent ductus arteriosus. J Surg Res. 2017 Feb;208:1-9.

5. Sato S, Miyake T, Kataoka M, Isoda K, Yazaki T, Tobita H, Ishimura N, Kinoshita Y. Efficacy of Repeated Lusutrombopag Administration for Thrombocytopenia in a Patient Scheduled for Invasive Hepatocellular Carcinoma Treatment. Intern Med. 2017 Sep 25.

6. Faella KH, Whiting D, Fynn-Thompson F, Matte GS. Bivalirudin Anticoagulation for a Pediatric Patient with Heparin-Induced Thrombocytopenia and Thrombosis Requiring Cardiopulmonary Bypass for Ventricular Assist Device Placement. J Extra Corpor Technol. 2016 Mar; 48(1):39-42.

7. Khalid S, Daw H. The Role of Apixaban in the Treatment of Heparin-Induced Thrombocytopenia. Cureus. 2017 Jul 5; 9(7):e1428.

8. Consolini R, Costagliola G, Spatafora D. The Centenary of Immune Thrombocytopenia-Part 2: Revising Diagnostic and Therapeutic Approach. Front Pediatr. 2017 Aug;21:5:179.

9. Luis Miguel JS, Juan José GF. Successful Long-Term Use of Eltrombopag in a Patient with Refractory Severe Thrombocytopenia Associated with Chronic Lymphocytic Leukemia That Allowed Oral Anticoagulant Treatment for Severe Cardiomyopathy. Case Rep Hematol. 2017; 2017:9538920.

\section{Figures}




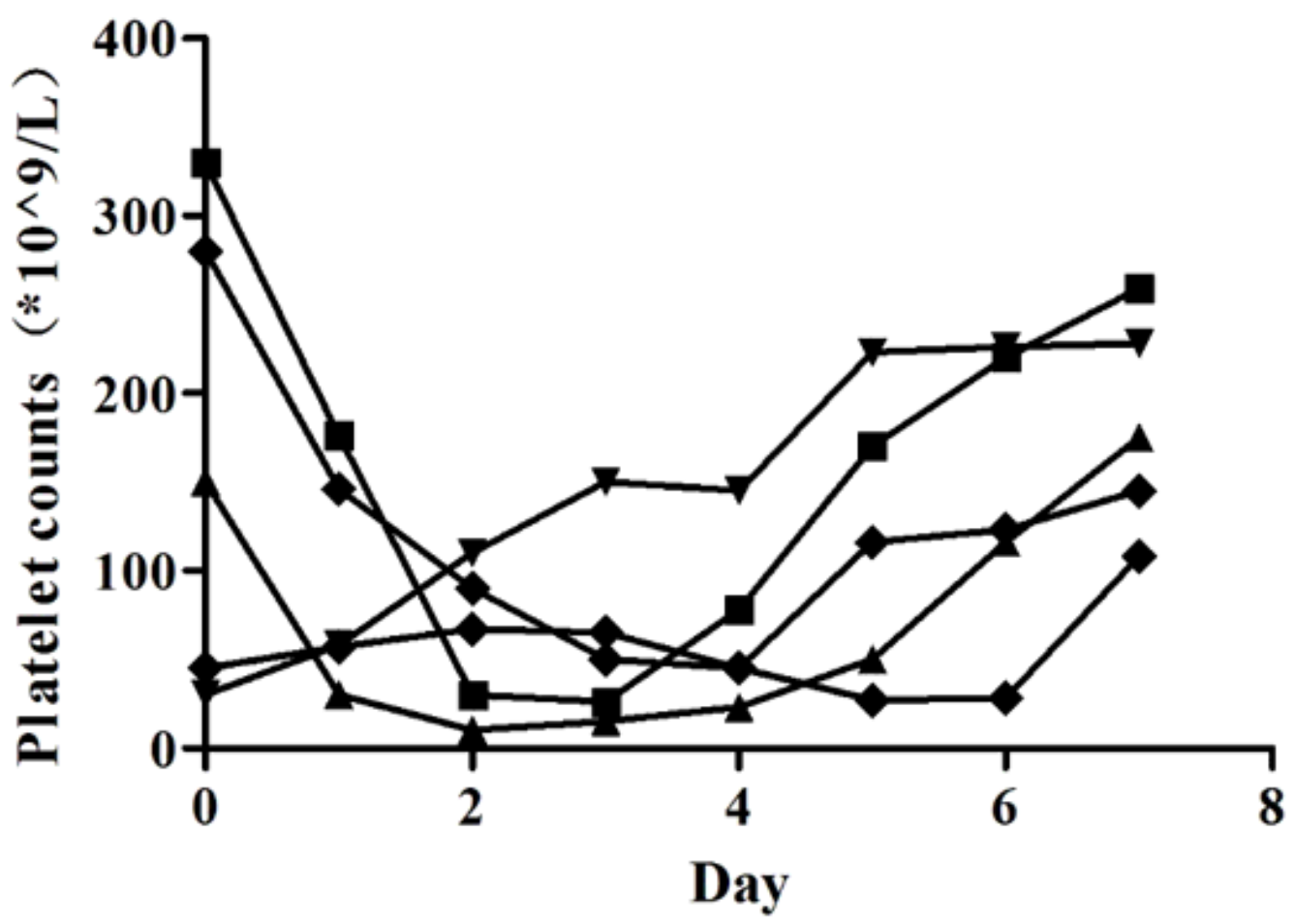

$\multimap$ Patient A

$\rightarrow$ Patient B

$\rightarrow$ Patient C

$\rightarrow$ Patient D

$\neg$ Patient E

Figure 1

Platelet counts in the 8 days after transcatheter CHD closure 


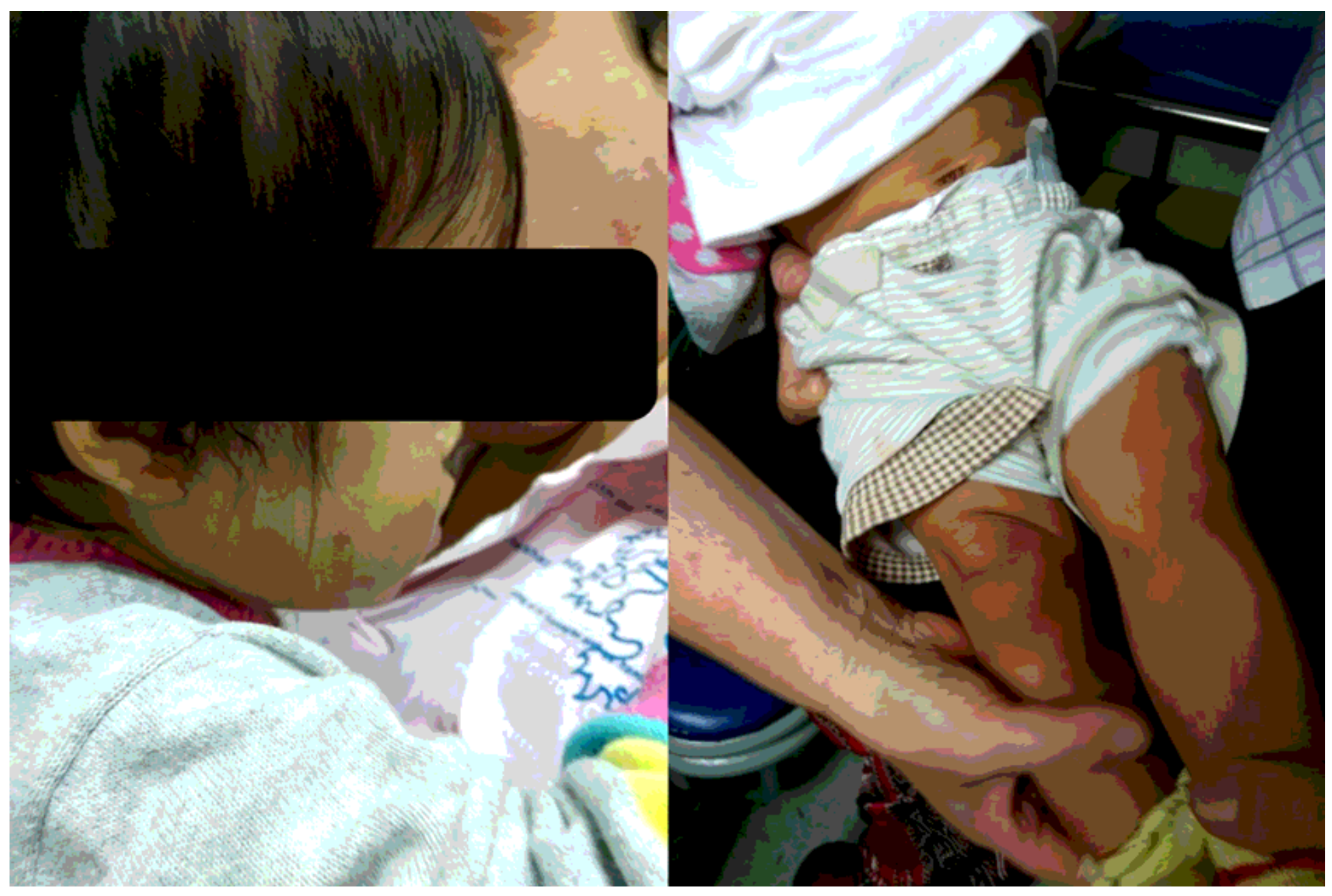

Figure 2

Ecchymosis scattered in the face and lower limbs 